\title{
Casual Interactions between Blood Pressure and Cardiac Inter-beat Intervals in Older People with Orthostatic Intolerance.
}

\author{
Marcos Hortelano $^{1}$, Richard B Reilly ${ }^{2}$, Raquel Cervigón ${ }^{1}$ \\ ${ }^{1}$ Universidad de Castilla-La Mancha, Cuenca, Spain \\ ${ }^{2}$ School of Engineering and School of Medicine. Trinity College Dublin, Republic of Ireland
}

\begin{abstract}
The aim of our study was to analysed the proportion of both casual direction between inter-beat interval $(R-R)$ and non-invasive beat to beat blood pressure $(B P)$ during six minutes walking distance test between symptomatic and asymptomatic participants of orthostatic intolerance (OI). We analysed 65 older subjects, of whom 42 were women. The participants underwent a supine to stand orthostatic test with non-invasive beat-to-beat systolic blood pressure and diastolic blood pressure and $R-R$ monitoring. Transfer Entropy (TE) method was applied to identify relationship between hemodynamical variables. The directionality index quantifies the preferred direction of information flow from difference between both directional couplings. Results show a higher symmetric bidirectional couplings in asymptomatic OI group. These results show that systolic blood pressure affects heart rate; nevertheless directional coupling between these cardiovascular signals is altered depending on changes in autonomic control introduced by the orthostatic test.
\end{abstract}

\section{Introduction}

Orthostatic hypotension $(\mathrm{OH})$ is a frequent cause of Orthostatic Intolerance (OI) and related symptoms associated with the occurrence of syncope. The transition from the supine to the upright position requires a reorganization of the mechanisms of cardiovascular control that may lead to neurally mediated syncope [1]. Other studies have already shown interesting results on changes in the irregularity and irreversibility of systolic blood pressure (SBP) and heart rate (HR) in patients with $\mathrm{OI}$, showing different entropy measures and time irreversibility indices as efficient markers to detect changes in heart rate variability, elicited by orthostatic in older people [2-4]. The magnitude of HR changes in relations to blood pressure (BP) changes traditionally considered to be mediated by the simulation of baroreceptors is expressed as baroreflex sensitivity (BRS). BRS is usually defined as the changes of the pulse inter- val or inter-beat interval (R-R) associated with a change of SBP by $1 \mathrm{mmHg}[5,6]$.

Moreover, baroreflex function is usually assessed from spontaneous oscillations of blood pressure (BP) and heart rate (HR) assuming the unidirectional influence from $\mathrm{BP}$ to HR. However, the interaction of BP and HR is bidirectional, HR also influence in BP [7].

Transfer entropy, which is related to the concept of Granger causality [8], has been proposed to distinguish effectively driving and responding elements and to detect asymmetry in the interaction of the different hemodynamic measurements $[8,9]$.Thus, the aim of our study was to analyse the changes in contribution of both causal directions between inter-beat interval (R-R), systolic blood pressure (SBP) and diastolic blood pressure (DBP) oscillations during six minutes walking distance test between symptomatic and asymptomatic participants of OI.

\section{Material}

The database included a total of 65 participants, aged over 70 years of age $(70.11 \pm 5.85)$, of whom $65 \%$ were females. There was no significant differences in age and gender between symptomatic and asymptomatic OI participants, $44.6 \%(n=29)$ had symptoms of OI and $55.4 \%$ $(n=36)$ did not.

Table 1. Database used to carry out our study.

\begin{tabular}{lccc}
\hline \hline & & Women & Men \\
\hline OI & Participants & 23 & 13 \\
& Age & $69.65 \pm 5.26$ & $71.77 \pm 6.47$ \\
\hline \multirow{2}{*}{ non-OI } & Participants & 19 & 10 \\
& Age & $70.63 \pm 5.34$ & $68.00 \pm 7.21$ \\
\hline \hline
\end{tabular}

The participants were evaluated in the Technology Research for Independent Living Clinic (TRIL) at St Jame's hospital in Dublin [10]. To carry out this study, the approval of the Local Research Ethics Commitee (SJH/AMNCH Research Ethics Commitee approval refer- 
ence number 13/06/2007) was necessary. Every participant got a minimum score of 23 in a Minimental state examination (MMSE), which is the threshold of insanity detection in the Irish framework [11]. All subjects consented before being included in this study. None of the participants suffered from Parkinson, diabetes mellitus, acute chronic renal failure, deficiency in B12 vitamin or a pacemaker. None of the participants took diurectics, ace inhibitor or angitotension II. A patient belonging to the older people group took beta blockers and another suffered diabetes. Likewise, the participants were not asked to stop taking their medication before the examination.

The measurement of the BP wave was carried out using the Finapres method (Finger Arterial Pressure). This method was developed to monitor BP steadily in a non-invasive way. It is composed of a finger band, a system of photoplethysmography and a unit to control pressure. This method enabled a reliable measurement of the BP to be taken beat to beat in a non-invasive way for the first time.

\section{Methods}

\subsection{Procedure}

In this study Transfer Entropy (TE) method was applied to identify relationship between hemodynamical variables. The 6 minutes walking distance test (6MWD) was applied, which is considered a protocol of simple effort, easy to do and which has proved to be a good reflection of the activities of a daily life. This test consists basically on measuring the distance which a person can walk in 6 minutes and it must be done along a long, flat an straight 30 meter long corridor. The 6MWD test is divided into clearly five identified and differentiated phases: a first phase of rest with a duration of 3 minutes, Phase 1 (pre-exercise). During the Phase 2 our participants begin the walk (starting of exercise). The Phase 3, with a duration of 6 minutes, in which participants are doing exercise (active phase). The Phase 4 which started when our participants ended the walk until they have recovered (recovery phase) and the Phase 5 at rest with a duration of 3 minutes (post-exercise). Due to this fact, we are going to work with a total of five phases (Phase 1, Phase 2, Phase 3, Phase 4, Phase 5). The same number of intervals was extracted in each of the five phases when the signal was stabilized ( $n=180$ samples) (Figure $1)$.

The following hemodynamic measurements beat by beat were registered:

- SBP: as the maximum blood pressure in the systole (mmHg).

- DBP: as the minimum value of pressure in the diastole (mmHg).

- CO: volume of blood expelled by each ventricle per minute (lpm).
- R-R: R-R interval in miliseconds (ms).

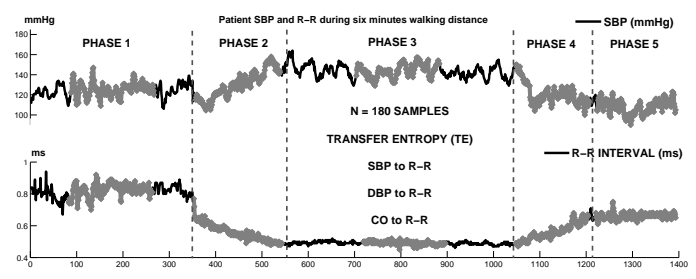

Figure 1. Patient SBP and R-R during six minutes walking distance.

\subsection{Transfer Entropy Methods}

We define symbols by reordering the amplitude values of time series $x_{i}$ and $y_{i}$. For a given, but otherwise arbitrary $i, m$ amplitude values $X_{i}=\{x(i), x(i+$ $l), \ldots, x(i+(m-1) l)\}$ are arranged in an ascending order $\left\{x\left(i+\left(k_{i 1}-1\right) l\right) \leq x\left(i+\left(k_{i 2}-1\right) l\right) \leq \ldots x\left(i+\left(k_{i m}-1\right) l\right\}\right.$, where $l$ denotes the time delay, and $m$ is the embedding dimension. In case of equal amplitude values the rearrangement is carried our according to the associated index $k$. A symbol is thus defined as $\bar{x}_{i}=\left(k_{i 1}, k_{i 2}, \ldots, k_{i m}\right)$, and with the relative frequency of symbols we estimate joint and conditional probabilities of the sequence of permutation indices [9].

Given symbol sequences $\bar{x}_{i}$ and $\bar{y}_{i}$ we define transfer entropy (TE):

$$
T_{Y, X}=\left(\bar{x}_{i+\delta}, \bar{x}_{i}, \bar{y}_{i}\right) \log \frac{p\left(\bar{x}_{i+\delta} \mid \bar{x}_{i}, \bar{y}_{i}\right)}{p\left(\bar{x}_{i+\delta} \mid \bar{x}_{i}\right)}
$$

where the sum runs over all symbols and $\delta$ denotes a time step.

- The directionality index $T=\left(T_{X, Y}-T_{Y, X}\right)$ quantifies the preferred direction of information flow.

- Positive values with $X$ as the driver.

- Negative values with $Y$ as the driver.

The directionality index from $S B P$ to $R-R, D B P$ to $R-R$ and $C O$ to $R-R$ were calculated. For symmetric bidirectional coupling we expect $T=0$.

\subsection{Statistical analysis}

An analysis of variance (ANOVA) was employed to test differences between phases and between groups for each hemodynamic parameter. Comparisons of continuous variables between the two groups were performed by Student's unpaired t-test or Mann-Whitney U test, where appropriate. Analysis of variance with repeated measurements ANOVA and with the Student-Newman-Keuls test were used to study the effect of one or more factors when at least one of them is a within-subjects factor or related 
measurements. When a significant overall effect was detected, Scheffe's F-test was used for comparison of the mean values for the two groups. The results were regarded as statistically significant for $\mathrm{p}<0.05$.

\section{4. $\quad$ Results}

The directionality index from SBP to R-R interval showed a greater symmetric bidirectional couplings in asymptomatic group with higher values of TE in symptomatic OI group in each of the phases (Table 2). However, those differences were only statistically significant in Phase $4(\mathrm{p}=0.042)$ (Figure 2).

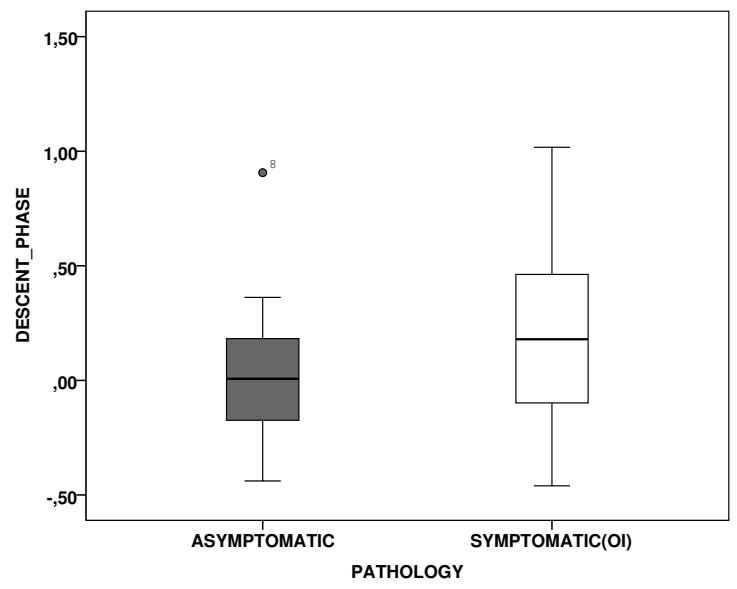

Figure 2. SBP to R-R Transfer Entropy in recovery phase.

In the same trend, the TE index from DBP to R-R interval showed a higher values in the symptomatic OI group (Table 2), thus the asymptomatic group illustrated a greater symmetric bidirectional couplings in all phases. Those differences were statistically significant in the Phase 4 $(\mathrm{p}=0.043)$, in the same way that the previous section (Figure 3 ).

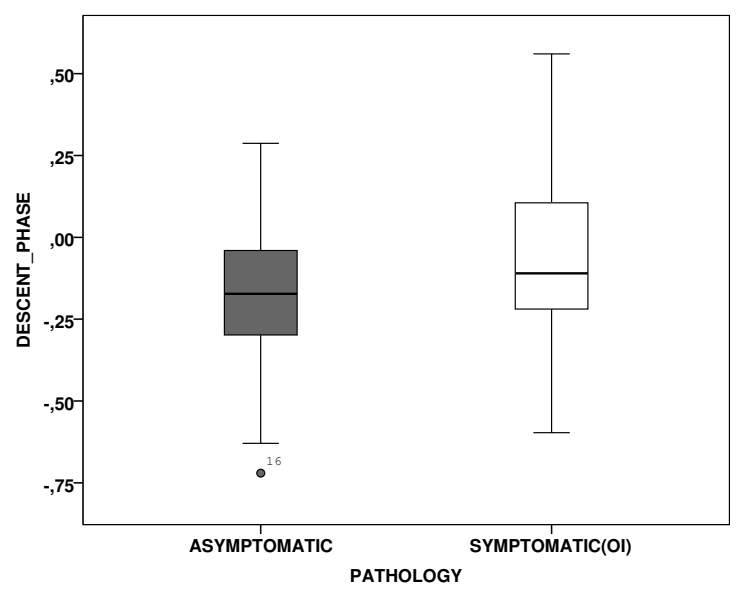

Figure 3. DBP to R-R Transfer Entropy in recovery phase.

On the other hand, we were able to observe that the directionality index from SBP to R-R showed positive values, being the SBP as a drive. Moreover, the index from DBP to R-R illustrated changes during active and passive phases in the preferred direction of flow information.

The index from $\mathrm{CO}$ to $\mathrm{R}-\mathrm{R}$ illustrated similar directionality trends that DBP to R-R, showing changes during active and passive phases in the preferred directions of flow information, but those differences did not reach statistically significance.

Table 2. Transfer Entropy results in Phase 4.

\begin{tabular}{ccccc}
\hline \hline TE & Phase & OI & non-OI & $(\mathrm{p})$ \\
\hline SBP - RR & Phase 4 & $0.19 \pm 0.36$ & $0.02 \pm 0.30$ & 0.042 \\
\hline DBP - RR & Phase 4 & $-0.07 \pm 0.26$ & $-0.19 \pm 0.23$ & 0.043 \\
\hline \hline
\end{tabular}

\section{Conclusion}

We assessed the proportion of both casual directions between R-R interval and beat to beat BP during six minutes walking distance test between symptomatic and asymptomatic participants of OI. The major finding of our study is that the directionality index of TE showed a greater symmetric bidirectional coupling in asymptomatic OI participants.

We were able to observe a decrease in the relationship between hemodynamic variables in symptomatic OI group, but those differences were only statistically significant in recovery phase (Figure 2) and (Figure 3). These results are coherent with previous studies, which showed that the drops in the information transfer from SBP to R-R and the directionality index suggested a loss of the baroreflex regulation of $\mathrm{HR}$ in the minutes preceding the decrease of pressure marking the beginning of syncope. Thus the disappearance of causality from SBP to R-R preceding the 
syncope event seems to forewarn the failure of cardiovascular regulation before the appearance of bradycardia and the fall in blood pressure that leads to fainting [1]. In the same way, other studies showed a significant decrease of the causal coherence from SBP to R-R evaluated, analyzed in the frequency domain an of the degree of nonlinear predictability of R-R given SBP in proximity of presyncope. Moreover, these results were complemented by the drop in the baroreflex gain during postural syncope [12-15]. Attention should also be drawn to results shown by others studies, where symptomatic OI patients illustrated a decrease in the time irreversibility indexes during active and passive stages [4]. Moreover, the directionality index from SBP to R-R illustrated SBP as a driver in the preferred direction of flow information. In addition, the directionality indexes from DBP to R-R interval showed changes during active and passive phases in the preferred direction of flow information. TE from $\mathrm{CO}$ to R-R showed the same trend, but those differences were not statistically significant. These results show that SBP affects HR; nevertheless directional coupling between these cardiovascular signals is altered depending on changes in autonomic control introduced by orthostatic test.

\section{Acknowledgements}

This work was supported by the Castilla-La Mancha Research Scheme (PPII- 20 14-024-P).

\section{References}

[1] Faes L, Nollo G, Porta A. Mechanisms of causal interaction between short-term rr interval and systolic arterial pressure oscillations during orthostatic challenge. Journal of Applied Physiology 2013;114(12):1657-1667.

[2] Hortelano M, Reilly RB, Cogan L, Cervigón R. Heart Rate Regularity Changes in Older People with Orthostatic Intolerance. Cham: Springer International Publishing. ISBN 978-3-319-16483-0, 2015; 337-346. URL http: / /dx.doi .org/10.1007/978-3-319-16483-034.

[3] Hortelano M, Reilly RB, Cervigón R. Multiscale entropy analysis of heart rate regularity changes in older people with orthostatic intolerance. In XIV Mediterranean Conference on Medical and Biological Engineering and Computing 2016. Springer, 2016; 62-66.

[4] Hortelano M, Reilly RB, Cervigon R. Multiscale time irreversibility to predict orthostatic intolerance in older people. In 2016 IEEE Statistical Signal Processing Workshop (SSP). June 2016; 1-5.

[5] Svacinová J, Javorka M, Nováková Z, Závodná E, Czip- pelová B, Honzíková N. Development of causal interactions between systolic blood pressure and inter-beat intervals in adolescents. Physiological Research 2015;64(6):821.

[6] BRISTOW JD, HONOUR AJ, PICKERING GW, SLEIGHT P, SMYTH HS. Diminished baroreflex sensitivity in high blood pressure. Circulation 1969; 39(1):48-54.

[7] Javorka M, Czippelova B, Chladekova L, Turianikova Z, Visnovcova Z, Lazarova Z, Tonhajzerova I. Causality of heart rate-blood pressure interactions during mental and orthostatic stress; .

[8] Granger CW. Investigating causal relations by econometric models and cross-spectral methods. Econometrica Journal of the Econometric Society 1969;424-438.

[9] Staniek M, Lehnertz K. Symbolic transfer entropy. Physical Review Letters 2008;100(15):158101.

[10] Kenny RA, Dishongh T, Newell F, Ni Scanail C. Research to reduce falls in older people: the tril centre. Geriatr Med 2009;39:326-327.

[11] Cullen B, Fahy S, Cunningham CJ, Coen RF, Bruce I, Greene E, Coakley D, Walsh JB, Lawlor BA. Screening for dementia in an irish community sample using mmse: a comparison of norm-adjusted versus fixed cut-points. International journal of geriatric psychiatry 2005;20(4):371376.

[12] Faes L, Widesott L, Del Greco M, Antolini R, Nollo G. Causal cross-spectral analysis of heart rate and blood pressure variability for describing the impairment of the cardiovascular control in neurally mediated syncope. IEEE transactions on biomedical engineering 2006;53(1):65-73.

[13] Iacoviello M, Forleo C, Guida P, Sorrentino S, D'Andria V, Rodio M, D'Alonzo L, Favale S. Independent role of reduced arterial baroreflex sensitivity during head-up tilt testing in predicting vasovagal syncope recurrence. Europace 2010;12(8):1149-1155.

[14] Mosqueda-Garcia R, Furlan R, Fernandez-Violante R, Desai T, Snell M, Jarai Z, Ananthram V, Robertson RM, Robertson D. Sympathetic and baroreceptor reflex function in neurally mediated syncope evoked by tilt. Journal of Clinical Investigation 1997;99(11):2736.

[15] Nollo G, Faes L, Antolini R, Porta A. Assessing causality in normal and impaired short-term cardiovascular regulation via nonlinear prediction methods. Philosophical Transactions of the Royal Society of London A Mathematical Physical and Engineering Sciences 2009;367(1892):1423-1440.

Address for correspondence:

Marcos Hortelano Rubio

Escuela Politecnica. Camino del Pozuelo sn, Cuenca. Spain

Marcos.Hortelano@uclm.es 